\title{
Article \\ Comprehensive Genome-Wide Identification and Transcript Profiling of GABA Pathway Gene Family in Apple (Malus domestica)
}

\author{
Qingbo Zheng ${ }^{1}$, Shenghui Su ${ }^{1}$, Zhe Wang ${ }^{2}$, Yongzhang Wang ${ }^{3}$ and Xiaozhao Xu ${ }^{1, *}$ \\ 1 College of Horticulture, Qingdao Agricultural University, Qingdao 266109, China; \\ qingbozheng@ibcas.ac.cn (Q.Z.); ssh8601@163.com (S.S.) \\ 2 Engineering Laboratory of Genetic Improvement of Horticultural Crops of Shandong Province, \\ Qingdao Agricultural University, Qingdao 266109, China; wangzhe19970502@163.com \\ 3 Laboratory of Quality \& Safety Risk Assessment for Fruit (Qingdao), Ministry of Agriculture and \\ Rural Affairs, Qingdao Agricultural University, Qingdao 266109, China; wangyzh304@163.com \\ * Correspondence: 201801006@qau.edu.cn; Tel.: +86-13280843966
}

Citation: Zheng, Q.; Su, S.; Wang, Z.; Wang, Y.; Xu, X. Comprehensive Genome-Wide Identification and Transcript Profiling of GABA Pathway Gene Family in Apple (Malus domestica). Genes 2021, 12, 1973. https://doi.org/10.3390/ genes12121973

Academic Editor: Mathilde Causse

Received: 11 October 2021

Accepted: 10 December 2021

Published: 12 December 2021

Publisher's Note: MDPI stays neutral with regard to jurisdictional claims in published maps and institutional affiliations.

Copyright: (c) 2021 by the authors. Licensee MDPI, Basel, Switzerland. This article is an open access article distributed under the terms and conditions of the Creative Commons Attribution (CC BY) license (https:/ / creativecommons.org/licenses/by/ $4.0 /)$.

\begin{abstract}
Aminobutyric Acid (GABA), a four-carbon non-protein amino acid, is a significant component of the free amino acid pool in most prokaryotic and eukaryotic organisms. GABA is involved in $\mathrm{pH}$ regulation, maintaining $\mathrm{C} / \mathrm{N}$ balance, plant development and defence, as well as a compatible osmolyte and an alternative pathway for glutamate utilization via anion flux. Glutamate decarboxylase (GAD, EC 4.1.1.15) and GABA transaminase (GABA-T, EC 2.6.1.19) are two key enzymes involved in the synthesis and metabolism of GABA. Recently, GABA transporters (GATs), protein and aluminium-activated malate transporter (ALMT) proteins which function as GABA receptors, have been shown to be involved in GABA regulation. However, there is no report on the characterization of apple GABA pathway genes. In this study, we performed a genome-wide analysis and expression profiling of the GABA pathway gene family in the apple genome. A total of 24 genes were identified including five GAD genes (namely MdGAD 1-5), two GABA-T genes (namely MdGABA-T 1,2), 10 GAT genes (namely GAT 1-10) and seven ALMT genes (namely MdALMT 1-7). These genes were randomly distributed on 12 chromosomes. Phylogenetic analyses grouped GABA shunt genes into three clusters-cluster I, cluster II, and cluster III-which had three, four, and five genes, respectively. The expression profile analysis revealed significant MdGAD4 expression levels in both fruit and flower organs, except pollen. However, there were no significant differences in the expression of other GABA shunt genes in different tissues. This work provides the first characterization of the GABA shunt gene family in apple and suggests their importance in apple response to abiotic stress. These results can serve as a guide for future studies on the understanding and functional characterization of these gene families.
\end{abstract}

Keywords: apple; GABA; transcript

\section{Introduction}

$\gamma$-Aminobutyric Acid (GABA) is a four-carbon amino acid; however, it is not an $\alpha$ amino acid and does not incorporate into proteins. GABA includes an amino group on the $\gamma$-carbon and is mostly found as a zwitterion, with both positive and negative electrical charges [1]. GABA is a flexible molecule substantial for many biological and physiological events such as inter and intracellular transformation, interaction with other biological signals [2,3], as well as the regulation of cell wall modification and gene expression [4-6].

In plants, numerous studies have revealed that GABA rapidly accumulates in response to biological and abiotic stresses [7], including temperature [8] (Zhu et al., 2019), osmotic pressure [6,9], and high salinity [10-12]. For example, the overexpression of GAD gene in Nicotiana sylvestris, application of exogenous GABA to muskmelon (Cucumis melo) and lettuce (Lactuca sativa) seedlings and GABA-T deficient in Arabidopsis have been shown 
to contribute to salt stress tolerance [6,11-14]. In addition, GABA has also been shown to regulate plant growth and development $[3,15]$. In tomato, the removal of the C-terminal autoinhibition region (calmodulin-binding domain) of $S I G A D$ increases GABA levels and wild type levels in fruits, and causes slow growth, more branched and shorter cortical parenchyma cell elongation and promoted fruit ripening [16]. Cell elongation was severely impaired in Arabidopsis pollen tubes, primary root, and hypocotyls when the GABA transaminase (GABA-T) gene was disrupted, leading to elevated tissue GABA concentrations $[5,11]$. In poplar, endogenous GABA accumulation interferes with hormone transport and carbon/nitrogen metabolism, which delays root primordia formation and inhibits root elongation $[17,18]$.

The synthesis and metabolic pathway of GABA have been referred to as GABA shunt associated with TCA, which is a feedback loop producing and conserving GABA supply $[11,19,20]$. In general, two pathways are involved in GABA synthesis and metabolism, where the main pathway starts with the decarboxylation of Glu to produce GABA and $\mathrm{CO}_{2}$ by the GAD enzyme in the cytosol [20]. The second pathway is catalyzed by GABA-T in a reversible transamination, which produces succinic semialdehyde (SSA). In addition, GABA, as a signal molecular, plays a variety of functions by transport and receptors of GABA. Aluminium-activated malate transporter (ALMT) proteins were first reported as a GABA receptor and activated by anions and negatively regulated by GABA in plant [15]. GABA exerts its multiple physiological effects in plants via ALMT, including the regulation of pollen tube and root growth, therefore GABA can finally be considered a legitimate signalling molecule in both the plant and animal kingdoms $[3,15,21]$.

GABA transporters (GATs), which belong to six of the amino acid/auxin permease (AAAP) subfamilies [22], mainly transport the ubiquitous amino acid across cellular membranes in flowering plants and play indispensable roles in various processes of plant growth and development [23]. LeProT1 is an efficient transporter for glycine betaine and the stress-induced GABA in tomato [24]. AtGAT1, the first high affinity GABA transporter protein, was reported in Arabidopsis. Characterization in heterologous expression systems showed that the kinetic properties and substrate selectivity of AtGAT1 are similar but distinct from mammalian, bacterial, and Saccharomyces cerevisiae GABA transporters [25]. Consistent with a role in GABA uptake into cells, transient expression of AtGAT1/green fluorescent protein fusion proteins in tobacco protoplasts revealed localization at the plasma membrane [25]. PeuGAT3 increased the thickness of xylem cells walls in both Arabidopsis and poplar and enhanced the lignin content of xylem tissues and the proline accumulation in poplar leaves, all of which may improve tolerance of salt/drought stress in desert poplars [26]. In addition, more putative GAT-like proteins were identified in the genome of Populus species [26-28].

The domesticated apple (Malus $\times$ domestica Borkh) is one of the most important fruit crops, widely distributed in temperate regions of the world. Recently, the genome of the domesticated apple was fully sequenced based on the doubled haploid GDDH13 [29,30]. This information provides an opportunity to further analyze the GABA pathway gene family in apple.

In this study, we identified 24 GABA pathway genes in the apple genome and investigated their phylogenetic relationships. Furthermore, the expression pattern and subcellular localization of the 24 genes were also investigated. Our results reveal the molecular characteristics and evolutionary pattern of the GABA pathway gene family and provide a foundation for future elucidation of the biological functions of GABA pathway genes in apples.

\section{Materials and Methods}

2.1. Identification and Classification of the Apple GABA Pathway Genes

We downloaded the apple genome data (GDDH13) from the Genome Database for Rosaceae (GDR, https: / / www.rosaceae.org/, 10 September 2020). Protein sequences of the Arabidopsis GABA pathway proteins were obtained from The Arabidopsis Information 
Resource (TAIR, https:/ /www.arabidopsis.org/, 9 October 2020). Apple GABA pathway genes were searched using the two basic local alignment search tool (BLAST) methods to identify the maximum number of GABA pathway genes. Then, primary GABA pathway proteins were identified using blast methods in UniProKB/Swiss-Prot database and the conserved domains were searched using CD-search on the NCBI website (https:/ / www. ncbi.nlm.nih.gov/Structure/cdd/wrpsb.cgi, 11 October 2020).

\subsection{Phylogenetic Analysis of the GABA Pathway Proteins}

The phylogenetic tree was constructed using Arabidopsis and apple GABA pathway full length protein sequences in MEGA7.0 (Arizona State University, Tempe, AZ, USA) with the Maximum Likelihood (ML) method with the following parameters: 1000 bootstrap test replicates, Jones-Taylor-Thornton (JTT) model, Uniform rates, Complete deletion, and Nearest-Neighbor-Interchange (NNI).

\subsection{Chromosomal Distribution and Synteny Analysis}

All GABA pathway genes were mapped to apple chromosomes using Amazing Gene Location From GTF/GFF by TBtools (https: / / github.com/CJ-Chen/TBtools, 15 October 2020) [31]. Synteny blocks between Apple and Arabidopsis genomes as well as within the apple genome were determined by Quick MCScanX Wrapper and visualized by Dual Synteny Plotter in TBtools.

\subsection{Sequences Analysis}

Conserved motifs in GABA pathway proteins were analyzed using the MEME (http: //meme-suite.org/, 19 October 2020) web server [32] (Bailey et al., 2009). The structures of GABA pathway genes were determined using Amazing Optional Gene Viewer in TBtools. The 2000 bp sequences of the GABA pathway genes start site (ATG) from the Genome Database for Rosaceae (https: / / www.rosaceae.org/, 25 October 2020) were downloaded and the cis-elements of promoters were identified by PlantCARE [33].

To predict the subcellular localization of GABA pathway proteins, simply paste protein's amino acid sequence (single letter code) in the WoLF PSORT window below and click submit (https: / / www.genscript.com/wolf-psort.html?src=leftbar, 30 October 2020).

\subsection{GABA Pathway Genes Expression Profiles}

The expression profiles of GABA pathway genes were determined in Malus domestica gene expression atlas of various organs. RNA-seq data sets (SRA accession no. SRP125281, SRP102870, and SRP050139) were retrieved from NCBI SRA database (https:/ / www.ncbi. nlm.nih.gov/sra, 8 November 2020). The expression patterns of apple GABA pathway genes in fruit developmental stages and bud development were acquired from NCBI SRA database (SRA accession no. SRP018878, SRP034165, SRP099578). To investigate the expression profiles of apple GABA pathway genes in response to different stress treatment (Penicillium expansum, Venturia inaequalis, Apple Stem Grooving Virus, Iron deficiencies, and Phosphorus deficiencies), apple RNA-seq data sets (SRA accession no. SRP150975, SRP034943, and SRP018878) were retrieved from NCBI SRA database (https: / / www.ncbi.nlm.nih.gov/sra, 12 November 2020).

The analysis of RNA-seq data was according to previous method and the FPKM (Fragments Per Kilobase of exon model per Million mapped fragments) were used to estimate the gene expression level. The heatmap of apple GABA pathway genes was exhibited using TBtools [34].

\section{Results}

3.1. Genome-Wide Identification and Characterization of GABA Pathway Gene Family Members in Apple

In the apple genome, a total of 24 putative genes involving GABA biosynthesis, catabolism, transports and receptors were identified. They were designed as $M d G A D$, 
MdGABA-T, MdGAT and MdALMT, respectively, according to different physiological and biochemical properties (Table 1). In addition, 24 putative genes sequence data can be found in Table S1. Detailly, five putative glutamate decarboxylases namely MdGAD that catalyzed glutamate conversion to produce GABA were identified. The length of the MdGAD proteins ranged from 333 to 510 amino acids, with predicted molecular weights of $37.68 \mathrm{kDa}$ to $56.95 \mathrm{kDa}$. The theoretical isoelectric point (pI) of the MdGAD proteins ranged from 4.95 to 5.71, and MdGAD1-4 protein subcellular localization predicted in the cytoplasmic, and MdGAD5 was predicted in the chloroplast; two putative GABA transaminase namely $M d G A B A-T$ genes that catalyzed GABA conversion to produce succinic semialdehyde (SSA) were identified. The length of the two MdGABA-T proteins was 514 and 521 amino acids, with predicted molecular weights of $56.69 \mathrm{kDa}$ and $57.59 \mathrm{kDa}$. The theoretical isoelectric point (pI) of the two MdGABA-T proteins was same 6.95, and MdGABA-T protein subcellular localization predicted in the chloroplast; 10 putative GABA transporters namely MdGAT genes that transport GABA from cell to cell were identified. The length of the MdGAT proteins ranged from 174 to 510 amino acids, with predicted molecular weights of $19.42 \mathrm{kDa}$ to $56.40 \mathrm{kDa}$. The theoretical isoelectric point (pI) of the MdGAT proteins ranged from 4.79 to 9.88 , and MdGAT protein subcellular localization predicted in the cytoplasmic, chloroplast, vacuole, and inner membrane; Aluminum-activated malate transporters (ALMTs) play an important role in aluminum tolerance, stomatal opening, and fruit acidity in plants. A total of $25 M d A L M T$ genes were identified from the apple reference genome of the "Golden Delicious" doubled-haploid tree (GDDH13) (Figure S1a) [35]. According to previous studies, ALMT proteins, which is the presence of a putative GABA-binding motif, are key transducers of GABA signaling in plants [15,36,37], Based on a putative GABA-binding motif, 12 amino acids in length shared between ALMTs participated in GABA signaling in plants [15]. Seven putative GABA receptors genes, namely MdALMT1, MdALMT2, MdALMT4, MdALMT5, MdALMT14, MdALMT17, and MdALMT20 that may play core roles in GABA signal transduction were identified in the apple genome (Table 1), which is closely related to TaALMT1 through the phylogenetic tree (Figure S1b) and contained the putative conserved GABA-binding motif (Figure S1c). The length of the seven putative GABA receptors MdALMT proteins ranged from 472 to 493 amino acids, with predicted molecular weights of $51.81 \mathrm{kDa}$ to $53.85 \mathrm{kDa}$. The theoretical isoelectric point (pI) of the seven MdALMT proteins ranged from 8.21 to 9.39, and all the seven MdALMT protein subcellular localizations were predicted in the inner membrane (Table 1).

\subsection{Chromosome Distribution and Duplication of Apple GABA Shunt Gene Family}

The 24 GABA pathway genes were widely distributed in 11 chromosomes of the apple genome (Figure 1). Of these genes, five $M d G A D$ genes were located on four chromosomes $(6,9,14$ and 16); two GABA-T genes were located on two chromosomes (9 and 17); $10 M d G A T$ genes were located on seven chromosomes (2, 3, 6, 7, 10, 11 and 14); and seven MdALMT genes were located on four chromosomes (0,3,11, 12 and 14). More GABA pathway genes were detected on chromosome 3,7, 9, 11 and 14; two genes each on chromosomes 0 and 6 , and gene on each of the remaining chromosomes.

It has been confirmed that whole-genome duplication and segmental duplication occurred during the process of apple domestication [30,38] (Jung et al., 2019; Velasco et al., 2010). Subsequently, whole-genome duplications and segmental duplications (WGD/segmental duplication) of the GABA pathway genes were analyzed (Figure 2). A total of $19(79 \%)$ GABA pathway genes, including three $M d G A D$ gene, two $M d G A B A-T$ gene, nine $M d G A T$ gene and five $M d A L M T$ gene in apple exhibited WGD/segmental duplication. These observations suggest that WGD/segmental duplication played an important role in the expansion of the apple GABA pathway genes family, as this process allowed the retention of numerous duplicated genes in the genome. 
Table 1. GABA pathway genes identified in this study. (cyto: cytoplasmic; chlo: chloroplast; plas: integral membrane; vacu: vacuole).

\begin{tabular}{|c|c|c|c|c|c|c|c|c|c|c|c|}
\hline Gene Name & $\begin{array}{l}\text { Gene Name } \\
\text { Abbreviation }\end{array}$ & Locus & Chromosome & $\begin{array}{c}\text { Start } \\
\text { Position }\end{array}$ & $\begin{array}{c}\text { End } \\
\text { Position }\end{array}$ & Strand & $\begin{array}{l}\text { Length } \\
\text { (aa) }\end{array}$ & pI & MW (kD) & $\begin{array}{c}\text { Subcellular } \\
\text { Localization } \\
\text { Predicted }\end{array}$ & $\begin{array}{l}\text { Potential } \\
\text { Functions }\end{array}$ \\
\hline \multirow{5}{*}{$\begin{array}{c}\text { Putative } \\
\text { glutamate } \\
\text { decarboxylase }\end{array}$} & $M d G A D 1$ & MD06G1235500 & Chr06 & 36665970 & 36669767 & - & 505 & 5.49 & 56.50 & cyto & \multirow{5}{*}{$\begin{array}{c}\text { GABA } \\
\text { Biosynthesis }\end{array}$} \\
\hline & $M d G A D 2$ & MD09G1276300 & Chr09 & 35359458 & 35366901 & - & 501 & 5.62 & 56.93 & cyto & \\
\hline & $M d G A D 3$ & MD09G1277000 & Chr09 & 35381876 & 35388878 & - & 501 & 5.71 & 56.85 & cyto & \\
\hline & $M d G A D 4$ & MD14G1242700 & Chr14 & 32108140 & 32110957 & - & 333 & 4.95 & 37.68 & cyto & \\
\hline & $M d G A D 5$ & MD16G1010800 & Chr16 & 826461 & 829289 & + & 510 & 5.65 & 56.95 & chlo & \\
\hline \multirow{2}{*}{$\begin{array}{c}\text { Putative GABA } \\
\text { transaminase }\end{array}$} & $M d G A B A-T 1$ & MD09G1139200 & Chr09 & 10792690 & 10798780 & - & 521 & 6.95 & 57.59 & chlo & \multirow{2}{*}{$\begin{array}{c}\text { GABA } \\
\text { Catabolism } \\
\end{array}$} \\
\hline & $M d G A B A-T 2$ & MD17G1128700 & Chr17 & 11307951 & 11313609 & - & 514 & 6.95 & 56.69 & chlo & \\
\hline \multirow{10}{*}{$\begin{array}{l}\text { Putative GABA } \\
\text { transporters }\end{array}$} & MdGAT1 & MD02G1244800 & Chr02 & 29475795 & 29480278 & - & 508 & 9.07 & 56.22 & plas & \multirow{10}{*}{$\begin{array}{l}\text { GABA } \\
\text { Transport }\end{array}$} \\
\hline & MdGAT2 & MD03G1133300 & Chr03 & 13280438 & 13300479 & - & 510 & 9.28 & 56.40 & plas & \\
\hline & $M d G A T 3$ & MD06G1103100 & Chr06 & 23991060 & 23995998 & + & 452 & 8.91 & 49.64 & cyto & \\
\hline & MdGAT4 & MD07G1072100 & Chr07 & 6824513 & 6827647 & + & 460 & 8.75 & 50.35 & plas & \\
\hline & MdGAT5 & MD07G1072200 & Chr07 & 6832373 & 6833039 & + & 174 & 4.79 & 19.42 & cyto & \\
\hline & MdGAT6 & MD07G1072300 & Chr07 & 6833186 & 6834458 & + & 299 & 9.22 & 32.55 & chlo & \\
\hline & MdGAT7 & MD10G1191600 & Chr10 & 28831972 & 28834442 & + & 457 & 8.97 & 50.79 & plas & \\
\hline & $M d G A T 8$ & MD11G1155800 & Chr11 & 14931465 & 14932148 & - & 177 & 9.88 & 19.42 & vacu & \\
\hline & MdGAT9 & MD11G1156000 & Chr11 & 14949839 & 14952668 & - & 202 & 7.69 & 22.50 & plas & \\
\hline & MdGAT10 & MD14G1122000 & Chr14 & 19672859 & 19676484 & + & 452 & 8.97 & 49.81 & plas & \\
\hline \multirow{5}{*}{$\begin{array}{l}\text { Putative GABA } \\
\text { receptors }\end{array}$} & $M d A L M T 4$ & MD03G1155200 & Chr03 & 17835181 & 17837669 & + & 476 & 8.73 & 52.20 & plas & \multirow{5}{*}{$\begin{array}{c}\text { GABA } \\
\text { Receptors }\end{array}$} \\
\hline & $M d A L M T 5$ & MD03G1155400 & Chr03 & 18018465 & 18020999 & + & 484 & 8.83 & 53.34 & plas & \\
\hline & MdALMT14 & MD11G1173000 & Chr11 & 19922876 & 19925870 & + & 485 & 9.39 & 53.75 & plas & \\
\hline & MdALMT17 & MD12G1040500 & Chr12 & 4405915 & 4408221 & - & 493 & 8.30 & 53.72 & plas & \\
\hline & $M d A L M T 20$ & MD14G1039500 & Chr14 & 3629641 & 3631853 & - & 494 & 8.84 & 53.85 & plas & \\
\hline
\end{tabular}




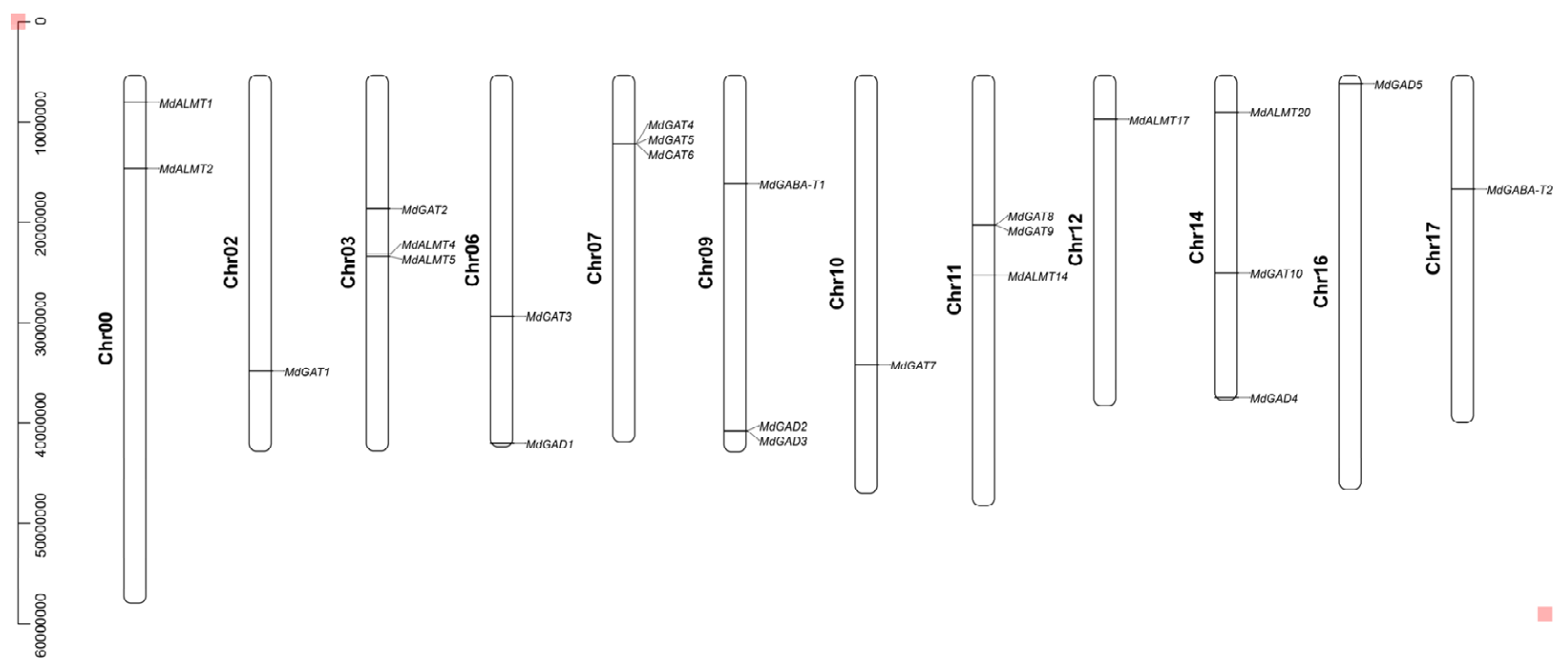

Figure 1. Chromosome distribution of the GABA pathway genes in apple.

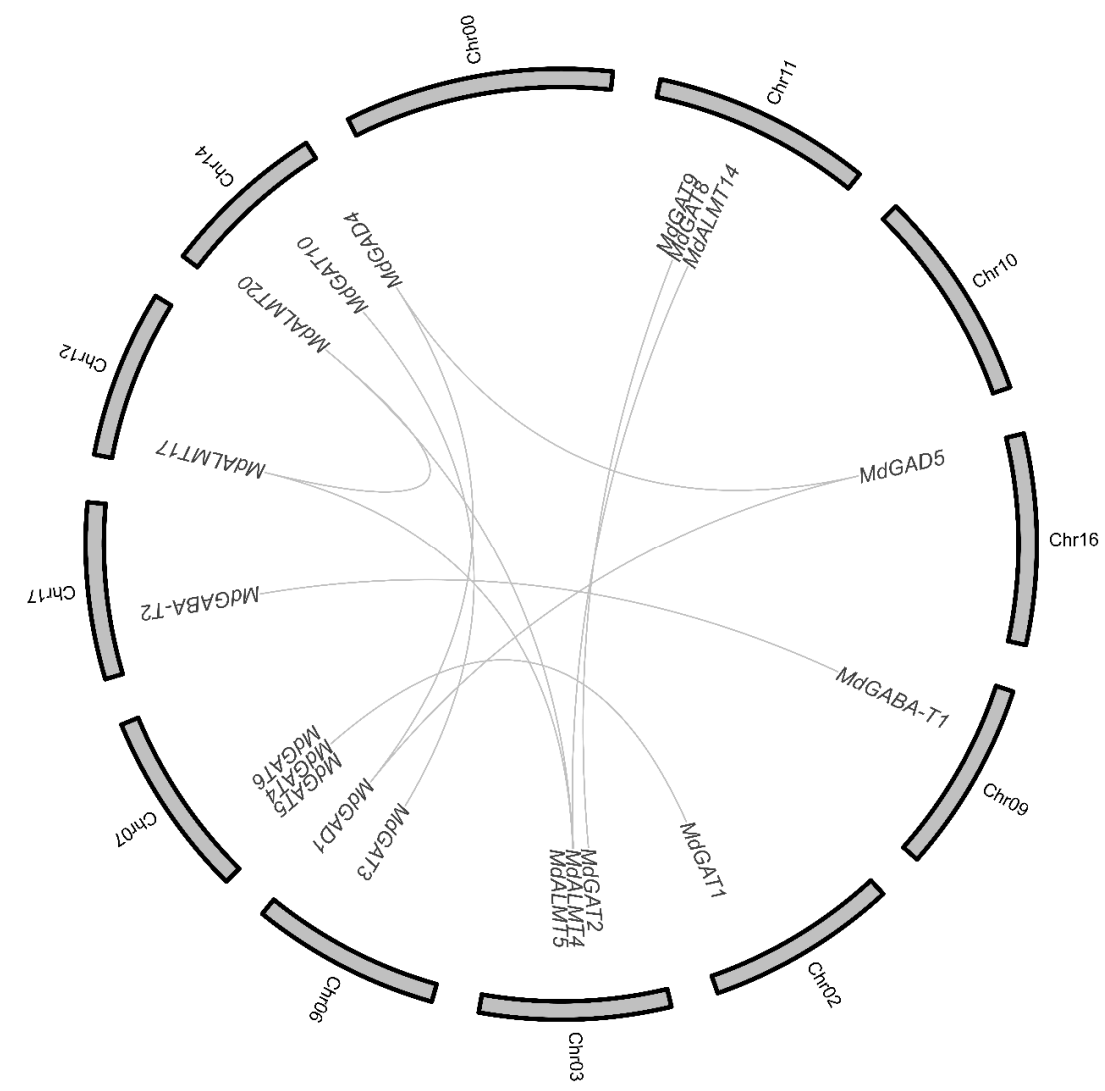

Figure 2. Collinearity analysis of GABA pathway genes in apple.

Ten pairs of paralogous GABA pathway genes were identified and distributed on different chromosomes in apple, whereas no tandem duplication events were observed, suggesting that segmental duplications were the main causes for the amplification of GABA pathway gene family. 
In addition, there are three genes involved in two segmental duplication events (MdALMT4/MdALMT17/MdALMT20).

\subsection{Evolutionary Relationships of GABA Shunt Genes between Apple and Arabidopsis}

To further explore the origin and evolutionary process of apple GABA shunt genes, we performed the comparative synteny map between apple and Arabidopsis genomes. According to comparative genomics, we can determine the origin and diversification of apple GABA shunt genes based on their Arabidopsis homologs. Large-scale syntenies contained 14 GABA shunt genes in apple and 9 GABA shunt genes in Arabidopsis (Figure 3 and Table S2). Among these syntenies, only one was unambiguous: MdGAD5-AT2G02000. More challenging for syntenic interpretation were cases where apple segmental duplications corresponded to a single Arabidopsis gene or where a single apple gene corresponded to multiple Arabidopsis genes. The first situation included MdGABA-T1/MdGABA-T2-AT3G22200, MdGAT2/MdGAT8-AT1G08230, MdGAT3/MdGAT10-AT5G41800, MdALMT4/MdALMT14AT2G27240, MdALMT17/MdALMT20-AT3G11680; whereas the second included MdGAD2AT1G65960/AT5G17330. Finally, a third case was identified where two duplicated apple genes corresponded to two Arabidopsis genes: MdGAD1/MdGAD4-AT2G02000/AT3G17720.

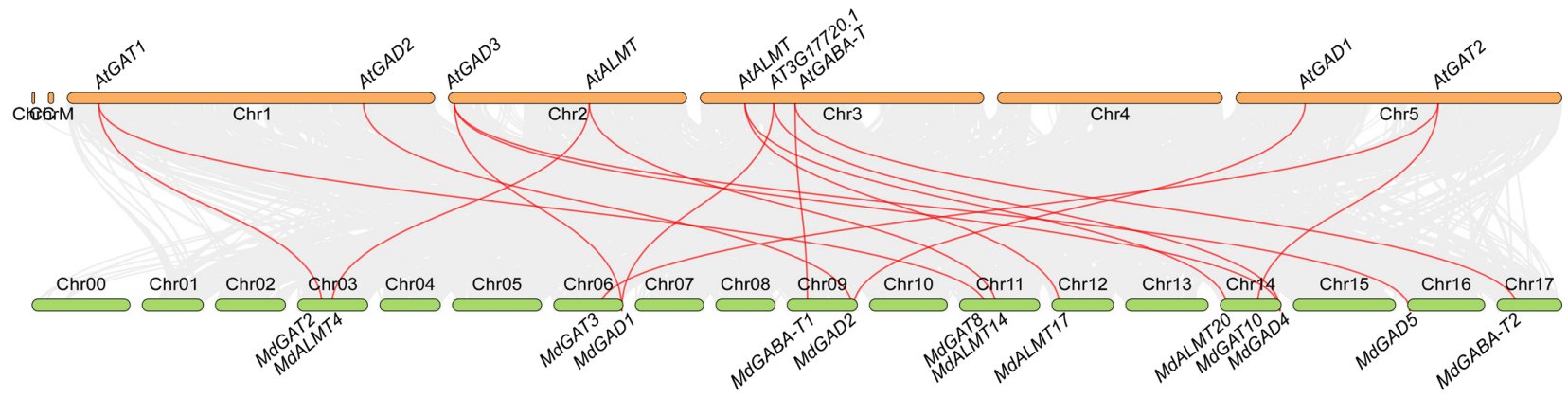

Figure 3. The comparative synteny map of GABA shunt genes between apple and Arabidopsis genomes.

\subsection{Gene Structure Analysis and Conserved Motif Identification in Apple GABA Pathway Genes}

To further study the conserved motif and structural features of the GABA pathway protein in apple, the gene structures, and conserved motifs of $M d G A D, M d G A B A-T, M d G A T$ and MdALMT were investigated. A total of 20 motif were identified in apple GABA pathway protein. Furthermore, MdGAD proteins have seven motifs, five of them were conserved; only one conserved motif displayed on MdGABA-T protein; eight motifs were found in MdGAT proteins, but none of them were conserved; nine motifs displayed on MdALMT and eight of them were conserved (Figure 4A). MdGAD, MdGABA-T, MdGAT and MdAMLT proteins demonstrated similar conserved motif composition, which was in agreement with the gene structure analysis. Specifically, the results showed that the highly conserved Glu-decarb-GAD domain existed in five MdGAD proteins; PLN02760 domain existed in two MdGABA-T proteins; SLC5-6-like-sbd domain was existed in two MdGAT proteins and ALMT domain existed in seven MdALMT proteins (Figure 4B). Almost all GABA pathway genes exhibited highly conserved exon-intron organization. Some special cases were observed; among five GAD gene family, GAD2 and GAD3 gene have a long intron, respectively. MdGAT2 gene, which was longest among seven $M d G A T$ family genes, has two long introns. In addition, the construction of MdALMT2 gene, which has one long intron, was different from the other six MdALMT gene (Figure $4 \mathrm{C}$ ). 
A

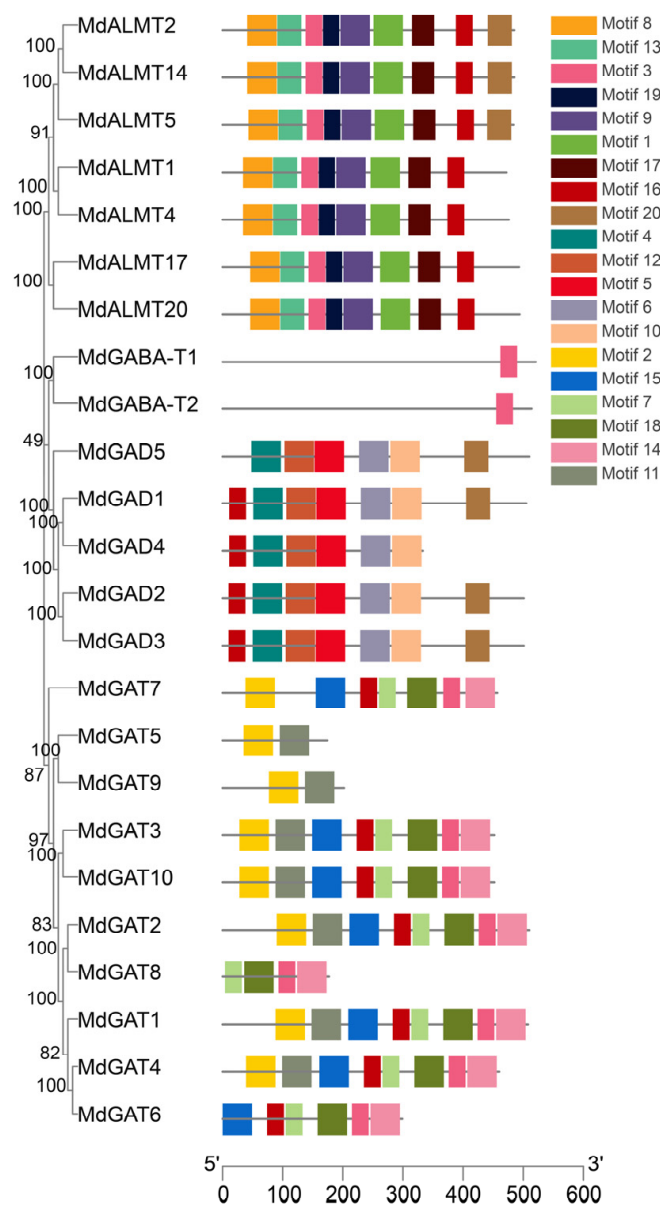

B

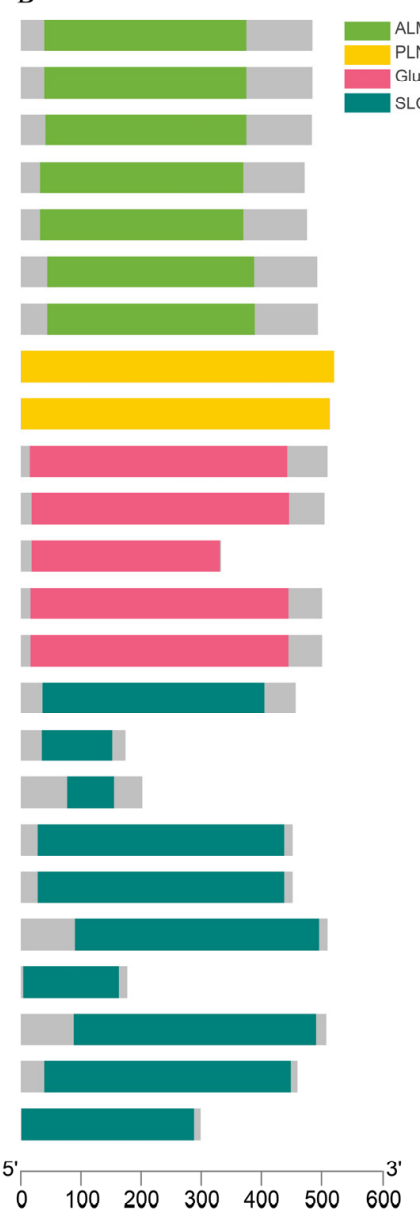

C

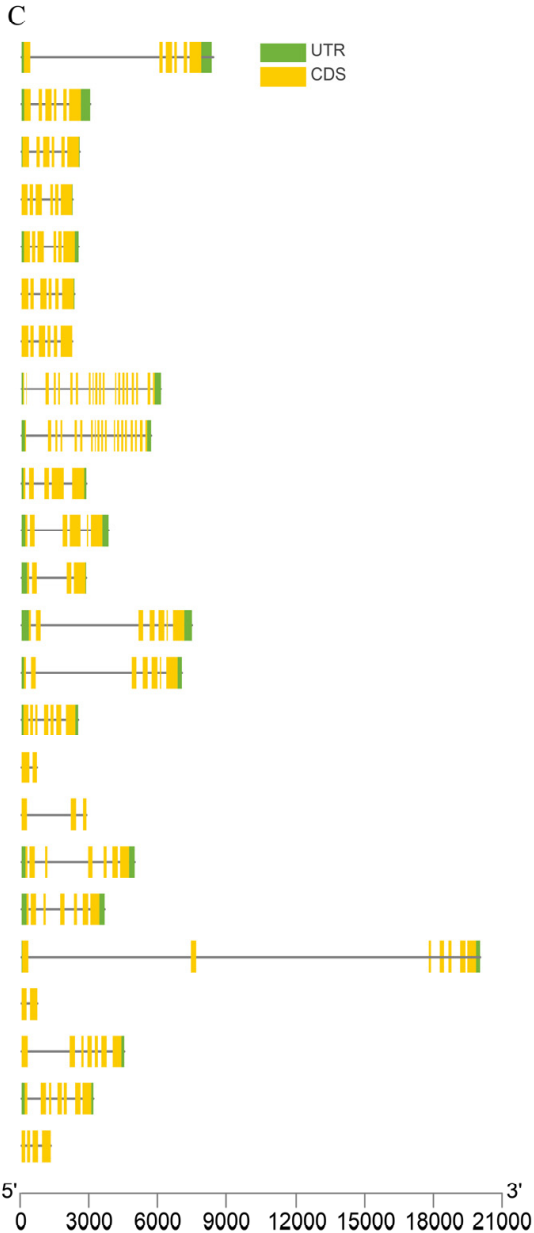

Figure 4. Phylogenetic relationships, motif composition, conserved domains, and gene structures of GABA pathway genes. (A) Conserved motifs analysis of MdGAD, MdGABA-T, MdGAT and MdAMLT proteins using MEME tools. Conserved motifs are showed in different colored boxes. (B) Functional domain analysis of MdGAD, MdGABA-T, MdGAT and MdAMLT proteins. (C) The intron/exon organization in GABA pathway genes is represented; yellow boxes depicting exons separated by introns with thin lines. Green boxes indicated UTRs.

3.5. Expression Profiles of GABA Pathway Genes in Different Tissues Development, Biological and Abiotic Stress

The tissue-specific expression of 24 GABA signal pathway genes was determined to compare their expression levels with stem, leaves, flower organ, fruit and seed tissues of apple through website information (http://bioinformatics.cau.edu.cn/AppleMDO, 15 November 2020) [39]. The results showed that (Figure 5 and Table S3), among 5 GAD genes, MdGAD4 was obviously expressed in leaves, shoot apex, flower organs, fruit, and seeds, especially, in petals, sepals and mature fruit peel. The expression level of MdGAD1 was higher in the shoot apex and flower organs and the expression level of MdGAD2 was obviously increased only in immature fruits, while the gene expression level of MdGAD3 and $M d G A D 5$ were not obviously expressed in different organ tissues. The MdGABA-T2 transcript level was higher in stem, flower, and fruit, but $M d G A B A-T 2$ transcript level was low in all tissues except for a slight increase in the receptacle and immature fruit. The expression of MdGAT and MdALMT family genes remained low level in all tissues except MdGAT1 and MdGAT1, which expression was obviously increased in the stem, flower organ, fruit, and seed tissues. These important indices concerning similarities and differences among the 17 tissue expressions suggested that MdGAD family genes may be involved in floral organ and fruit development. 


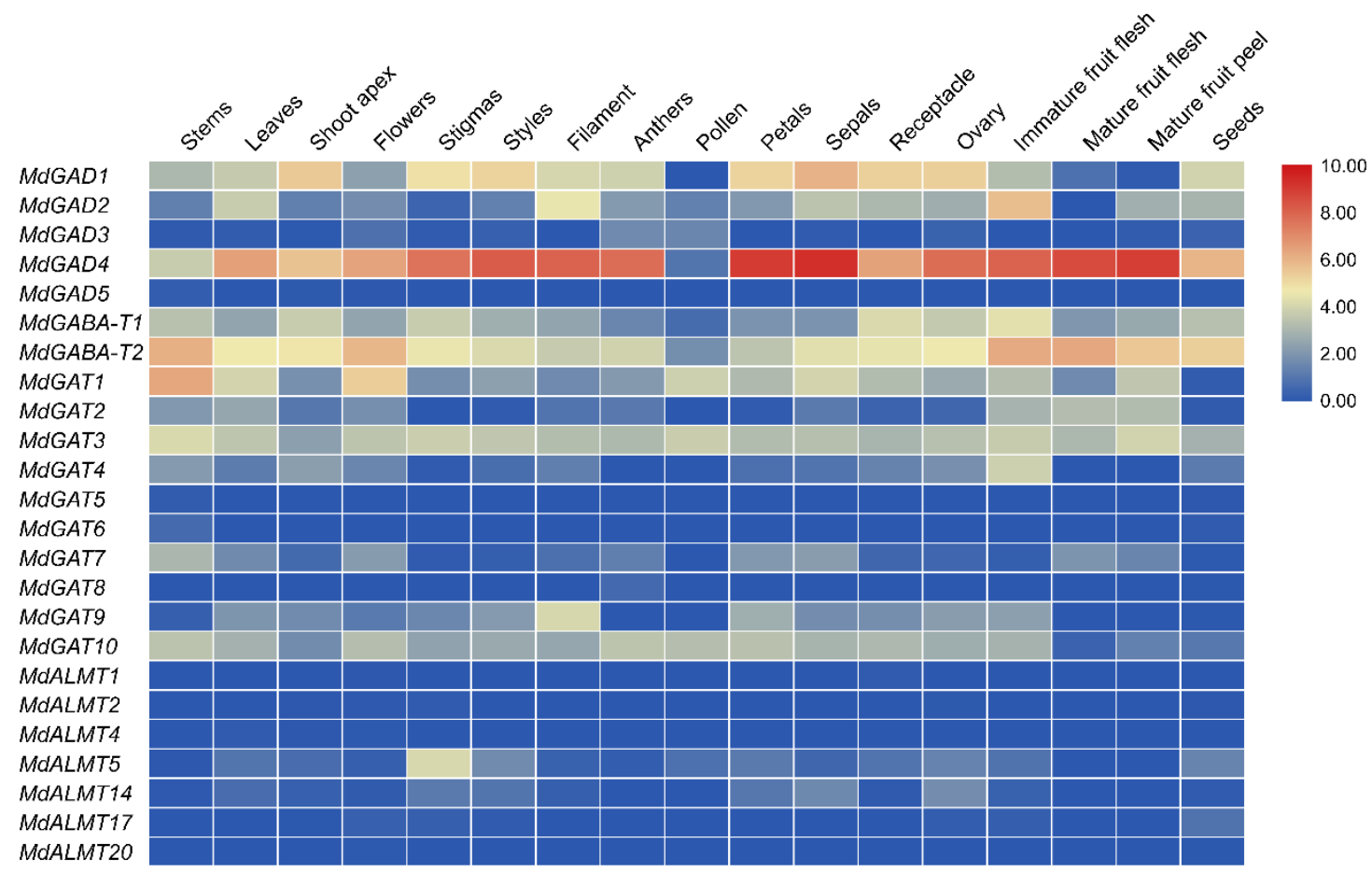

Figure 5. Heat map of GABA signal pathway genes in the stem, leaves, flower organ, fruit and seed tissues. Relative expression values with color blue (low) to red (high) are displayed at the bottom.

In addition, the expression of 24 GABA signal pathway gene involving the growth and developmental process of apple were investigated use the website (http:/ / bioinformatics. cau.edu.cn/AppleMDO, 15 November 2020) [39]. Meanwhile, the GABA signal pathway gene, which responded to several biological and abiotic stresses, was investigated in a previous study [40]. The result (Figure 6 and Table S4) showed that most of GABA signal pathway gene has no significant expression during the fruit development process, except for MdGAD1 and MdGAD4. Specifically, a slightly higher expression of MdGAD1 in the first and second week after full bloom was observed, which subsequently declined from the third to the twentieth week; the expression of MdGAD4 showed a slight increase at 87 days post-anthesis and twentieth week after full bloom. During buds' development and growth process, among 24 GABA signal pathway genes, the MdGAD1, MdGABA-T2 and MdAMLT5 showed a similar expression pattern with an increase and then, subsequently, a decline from initiation of dormant buds to bud break. Based on the results above, we further acknowledge that GABA maybe play an important role during fruit and buds' development.

The treatments of Apple Stem Grooving Virus (ASGV) infected iron and phosphorus deficiencies are commonly biological and abiotic stresses in plant development [40]. The expression of 24 GABA signal pathway genes has no significant difference in these treatments. Additionally, there is no obvious variation pattern of these genes after the treatment of leaves infected with $V$. inaequalis (VI). Unexpectedly, the expression of GABA pathway genes has significant variations in the treatment of fruit infected with $P$. expansum (PE). To be more specific, the expression of MdGAD2, MdGAD3 and MdGAD5 were significantly up regulated after fruit infected with PE. On the contrary, a significant down-regulation in the gene expression of MdGAD1 and MdGAD4 after fruit was infected with PE was observed. The up-regulation expression of $M d G A B A-T 1$ and the down-regulation expression of $M d G A B A-T 2$ after fruit infected with PE treatment was also observed. In view of the above results, we indicated that the GABA signal pathway gene plays a core role in floral organ, fruit and buds development, especially of the GAD and GABA-T gene family. 


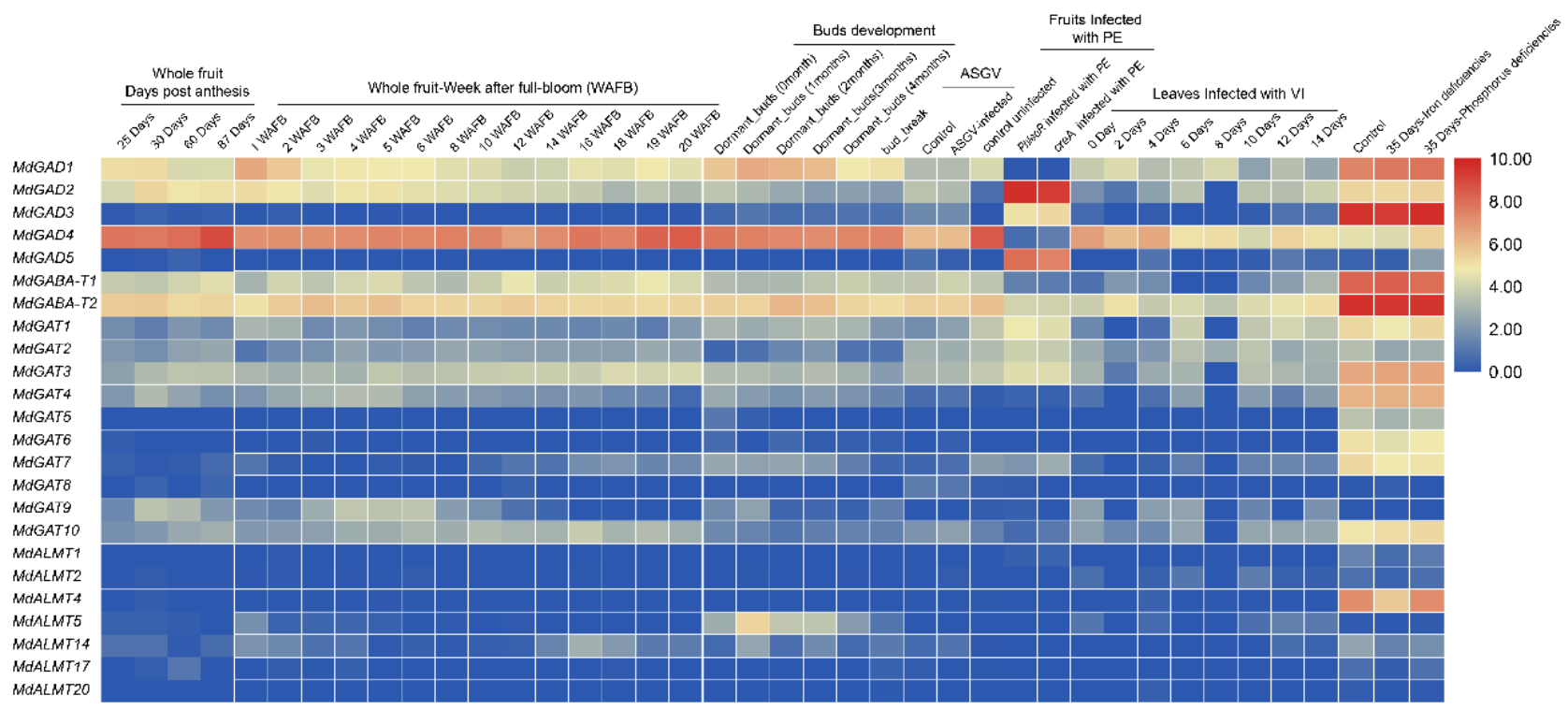

Figure 6. The expression of 24 GABA signal pathway gene involving growth and developmental process of apple were investigated use the website. Relative expression values with color blue (low) to red (high) are displayed at the bottom.

\subsection{Cis-Acting Elements in the Promoter of the GABA Pathway Genes}

For further insight into the genes function and regulation mechanism of GABA signal pathway, including MdGADs, MdGABA-Ts, MdGATs and MdALMTs, the cis-acting elements in promoter sequences were analyzed. The GABA signal pathway genes promoters, including $2000 \mathrm{bp}$ of genomic DNA sequence upstream of the translation starts site, were submitted in the PlantCARE database [33]. All cis-acting regulatory components for each single promoter sequence were identified (Figure 7 and Table S5).

In hormone-related cis-acting elements, the $\mathrm{ABA}$ responsive element (ABRE), the MeJA-responsive element (CGTCA-motif and TGACG-motif), the salicylic acid (SARE and TCA-element), and the ethylene-responsive element (ERF) were identified in the promoter region of the most of GABA pathway genes. Gibberellin-responsive element (P-box, GARE-motif, and TATC-box) and auxin-responsive element (AuxRR-core and TGAelement) were observed in the promoter of $M d G A B A-T, M d G A T$ and $M d A L M T$ genes, but none of MdGAD genes promoter was observed (Figure 7 and Table S5). Lots of hormoneresponsive elements were observed in the promoter region of GABA pathway genes, revealing a close relationship with GABA and hormones and also reconfirming that the GABA signal pathway could play important functions in the regulation of plant growth and development.

In stress-related cis-acting elements, anaerobic induction (ARE), defense and stress responsiveness (MBS), stress response (HSE) and low-temperature (LTR) responsiveness elements were also detected in the promoters of $M d G A D, M d G A B A-T, M d G A T$ and $M d A L M T$ genes. Meristem expression (CAT-box, CCGTCCbox and dOCT) and endosperm expression (GCN4_motif and Skn-1_motif) were found in the promoter of GABA signal pathway genes. Unexpectedly, the promoter of GABA signal pathway gene, which includes $M d G A D s, M d G A B A-T s, M d G A T s$ and MdAMLTs, was rich in the WRKY transcription factor binding site (Figure 7 and Table S5). 


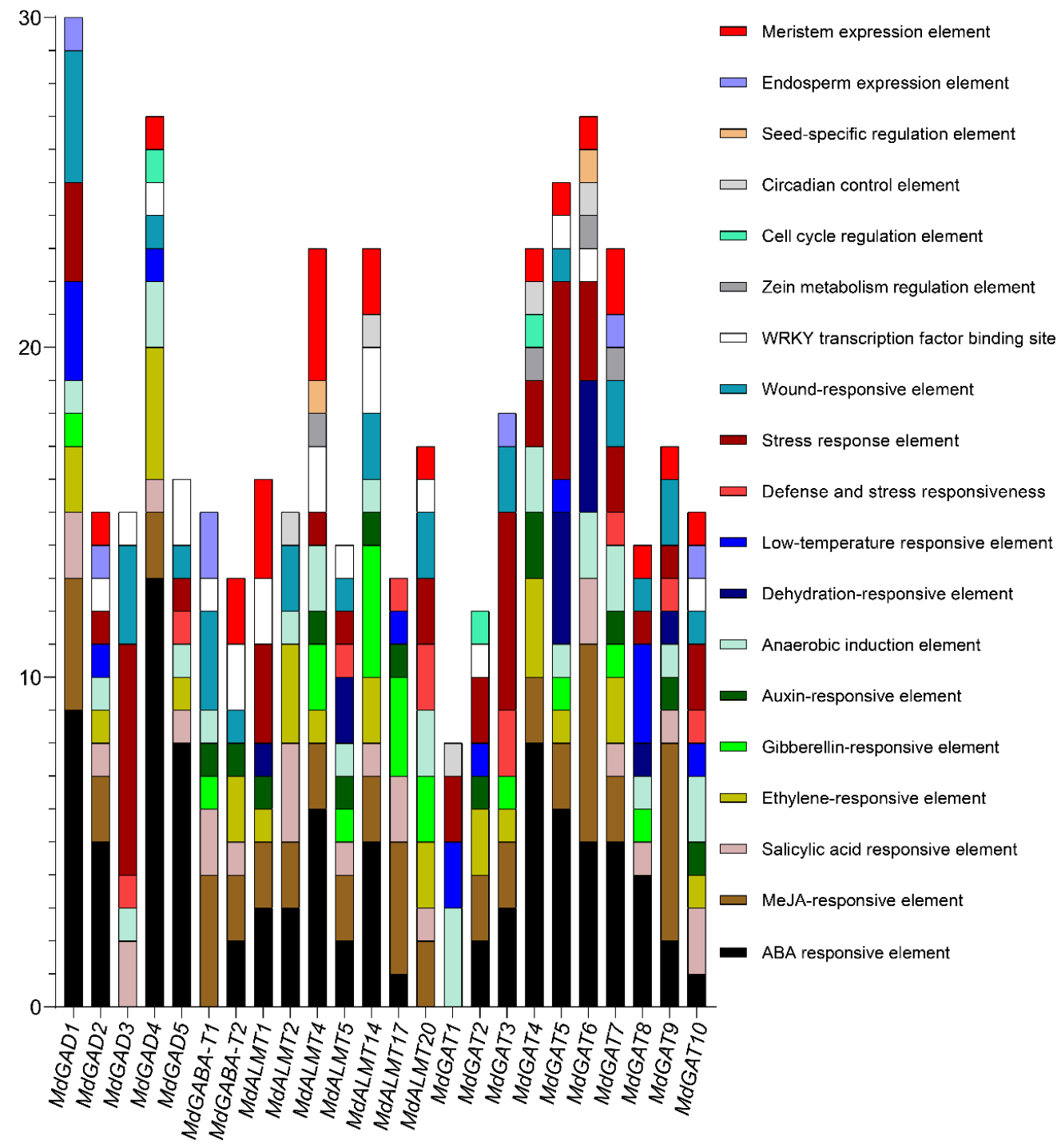

Figure 7. Illustration of cis-regulatory units in all promoters of the GABA signal pathway gene in apple. From the translation start site, the upstream region $2000 \mathrm{bp}$ of each GABA signal pathway genes promoter.

\section{Discussion}

GABA, as a signaling molecule, plays a crucial role in plant growth and development, biological and abiotic stresses, $\mathrm{pH}$ regulation and maintaining $\mathrm{C} / \mathrm{N}$ balance, etc. [3,5,6,41]. There have four types of GABA signal pathway genes, namely GAD, GABA-T, GAT and ALMT that are involved in the synthesis, metabolism, transport, and receptor of GABA, respectively. However, there are no reports on the characterization of apple GABA signal pathway genes despite the availability of the apple genome sequence. In this study, we performed a genome wide analysis and expression profiling of the GABA pathway gene family in the apple genome. These results can serve as a guide for future studies on the understanding and functional characterization of these gene families.

$\mathrm{GAD}$, an essential rate-limiting enzyme, catalyzes glutamate converting to GABA and is widely distributed in organisms [7,42]. In a previous study, the gene code GAD enzymes have been successfully identified by RACE method in many species, such as Arabidopsis [43], maize [44], and also in some woody plants, such as apple [45], citrus [42], tea [46] and Caragana intermedia [47]. However, a comprehensive analysis of the GAD gene by bioinformatics was not well understood. In addition, several studies have shown that different GAD gene family members have different expression patterns. For example, AtGAD1 is mainly expressed in Arabidopsis roots [43], while ZmGAD1 is expressed 
in the leaves, stems, and roots of maize [44]. In rice, OsGAD1 is mainly expressed in seeds, but OsGAD2 is mainly expressed in roots [48]. In citrus, CsGAD1 is predominantly expressed in flowers, but CsGAD2 is predominantly expressed in fruit [42]. These studies also indicated that GADs might play important roles in plant development and stress responses. In this current study, five GAD genes were identified in the apple genome, among the five GAD genes, MdGAD4 was obviously expressed in leaves, shoot apex, flower organs, fruit, and seeds, especially, in petals, sepals and mature fruit peel. Additionally, the expression level of $M d G A D 1$ was higher in the shoot apex and flower organs and the expression level of MdGAD2 was obviously increased only in immature fruits. For the developmental phase, the expression of $M d G A D 4$ was obviously higher in fruit and buds ${ }^{\prime}$ development than other $M d G A D$ family members (Figure 5). The reason for this might be that the promoters of the $M d G A D 1, M d G A D 2$ or $M d G A D 4$ contain more phytohormone, metabolism and development-related cis-elements than those of MdGAD3 or MdGAD5 (Figure 7). In addition, the five GADs perform differently in response to biological and abiotic stresses and the expression of $M d G A D 2, M d G A D 3$ and MdGAD5 were obviously up regulated after fruit infected with PE. On the contrary, a significant down-regulation in the gene expression of $M d G A D 1$ and MdGAD4 after fruit infected with PE was observed (Figure 6).

The efflux malate anions through channels are stimulated by external $\mathrm{Al}^{3+}$ ions. This feature of a few proteins determined the name of the entire protein family as Aluminumactivated Malate Transporters (ALMT) [49]. In an interesting study, TaALMT1 acting as a GABA receptor was first found in wheat, resulting in altered root growth and tolerance to $\mathrm{Al}$, acidic, or alkaline $\mathrm{pH}$ [15]. This mechanism would help the plant to avoid excessive loss of reduced carbon, which is crucial for plant growth and development under stress [50]. A total of $25 M d A L M T$ genes were identified from the apple reference genome of the "Golden Delicious" doubled-haploid tree (GDDH13) [35]. Based on GABA receptor characteristics with 12 amino acids in length, shared between ALMT and the ion channels used to construct the $\alpha_{1} \beta_{2} \gamma_{2}$ GABA $_{\mathrm{A}}[36,37]$, seven putative GABA receptors, namely MdALMT genes that play core roles in GABA signal transduction, were identified (Table 1 and Figure S1). In previous studies, phylogenetic analyses of ALMT proteins from plants, such as Arabidopsis thaliana, Populus trichocarpa, Oryza sativa, Selaginella moellendorffii, and moss Physcomitrella patens, subdivided these proteins into five distinct clades [3,51]. Clade 1, 2a, 3, 4, and 5 all have GABA motifs that can be predicted to be GABA sensitive, except clade $2 \mathrm{~b}$. The motif region from wheat (TaALMT1), barley (HvALMT1), rice (OsALMT5), and Arabidopsis (AtALMT1), which fall into the evolutionary clade 1, have been shown to be regulated by GABA [15].

Supplementary Materials: The following are available online at https:/ /www.mdpi.com/article/ 10.3390/genes12121973/s1, Figure S1: Analysis of gene and protein sequences structure and conserved motif of MdALMTs, Table S1: Promoter, CDS, and Protein sequences of GABA signaling pathway genes, Table S2: Synteny analysis of apple and Arabidopsis GABA signaling pathway genes, Table S3: FPKM of $M$. domestica GABA signaling pathway genes in the stem, leaves, flower organ, fruit and seed tissues, Table S4: FPKM of $M$. domestica GABA signaling pathway genes in different growth and developmental process, Table S5: Cis-elements in promoter region of M. domestica GABA signaling pathway genes.

Author Contributions: Conceptualization, Z.W., Y.W. and X.X.; methodology, Q.Z., S.S. and X.X.; software, X.X.; writing—original draft preparation, Q.Z. and X.X.; writing—review and editing, Q.Z. and X.X. All authors have read and agreed to the published version of the manuscript.

Funding: This research was funded by the National Natural Science Foundation of China (grant number 31801824), Specialist Project of Fruit Tree Innovation Team, Technical System of Modern Agricultural Industry in Shandong Province (SDAIT-06-05), and Scientific Research Funds for Highlevel Personnel of Qingdao Agricultural University (663/1118036).

Institutional Review Board Statement: Not applicable.

Informed Consent Statement: Not applicable. 
Data Availability Statement: Data is contained within the article or supplementary material, further inquiries can be directed to the corresponding author.

Conflicts of Interest: The authors declare no conflict of interest.

\section{References}

1. Seifikalhor, M.; Aliniaeifard, S.; Hassani, B.; Niknam, V.; Lastochkina, O. Diverse role of $\gamma$-aminobutyric acid in dynamic plant cell responses. Plant Cell Rep. 2019, 38, 847-867. [CrossRef]

2. Salah, A.; Zhan, M.; Cao, C.; Han, Y.; Ling, L.; Liu, Z.; Li, P.; Ye, M.; Jiang, Y. $\gamma$-Aminobutyric Acid Promotes Chloroplast Ultrastructure, Antioxidant Capacity, and Growth of Waterlogged Maize Seedlings. Sci. Rep. 2019, 9, 484. [CrossRef] [PubMed]

3. Ramesh, S.A.; Tyerman, S.D.; Gilliham, M.; Xu, B. $\gamma$-Aminobutyric acid (GABA) signalling in plants. Cell. Mol. Life Sci. 2017, 74, 1577-1603. [CrossRef] [PubMed]

4. Shelp, B.J.; Bown, A.W.; Faure, D. Extracellular $\gamma$-aminobutyrate mediates communication between plants and other organisms. Plant Physiol. 2006, 142, 1350-1352. [CrossRef] [PubMed]

5. Renault, H.; El Amrani, A.; Palanivelu, R.; Updegraff, E.P.; Yu, A.; Renou, J.P.; Preuss, D.; Bouchereau, A.; Deleu, C. GABA accumulation causes cell elongation defects and a decrease in expression of genes encoding secreted and cell wall-related proteins in Arabidopsis thaliana. Plant Cell Physiol. 2011, 52, 894-908. [CrossRef] [PubMed]

6. Renault, H.; El Amrani, A.; Berger, A.; Mouille, G.; Soubigou-Taconnat, L.; Bouchereau, A.; Deleu, C. $\gamma$-Aminobutyric acid transaminase deficiency impairs central carbon metabolism and leads to cell wall defects during salt stress in Arabidopsis roots. Plant Cell Environ. 2013, 36, 1009-1018. [CrossRef]

7. Shelp, B.; Bozzo, G.; Trobacher, C.; Chiu, G.; Bajwa, V. Strategies and tools for studying the metabolism and function of Y-aminobutyrate in plants. I. Pathway structure. Botany 2012, 90, 651-668. [CrossRef]

8. Zhu, X.; Liao, J.; Xia, X.; Xiong, F.; Li, Y.; Shen, J.; Wen, B.; Ma, Y.; Wang, Y.; Fang, W. Physiological and iTRAQ-based proteomic analyses reveal the function of exogenous $\gamma$-aminobutyric acid (GABA) in improving tea plant (Camellia sinensis L.) tolerance at cold temperature. BMC Plant Biol. 2019, 19, 43. [CrossRef]

9. Vijayakumari, K.; Puthur, J.T. $\gamma$-Aminobutyric acid (GABA) priming enhances the osmotic stress tolerance in Piper nigrum Linn plants subjected to PEG-induced stress. Plant Growth Regul. 2016, 78, 57-67. [CrossRef]

10. Xing, S.G.; Jun, Y.B.; Hau, Z.W.; Liang, L.Y. Higher accumulation of $\gamma$-aminobutyric acid induced by salt stress through stimulating the activity of diamine oxidases in Glycine max (L.) Merr. Roots. Plant Physiol. Bioch. 2007, 45, 560-566. [CrossRef]

11. Renault, H.; Roussel, V.; El Amrani, A.; Arzel, M.; Renault, D.; Bouchereau, A.; Deleu, C. The Arabidopsis pop2-1 mutant reveals the involvement of GABA transaminase in salt stress tolerance. BMC Plant Biol. 2010, 10, 20. [CrossRef]

12. Akcay, N.; Bor, M.; Karabudak, T.; Ozdemir, F.; Turkan, I. Contribution of Gamma amino butyric acid (GABA) to salt stress responses of Nicotiana sylvestris CMSII mutant and wild type plants. J. Plant Physiol. 2012, 169, 452-458. [CrossRef]

13. Xiang, L.X.; Hu, L.P.; Hu, X.H.; Pan, X.B.; Ren, W.Q. Response of reactive oxygen metabolism in melon chloroplasts to short-term salinity-alkalinity stress regulated by exogenous $\gamma$-aminobutyric acid. J. Ecol. 2015, 26, 3746-3752. (In Chinese)

14. Kalhor, M.S.; Aliniaeifard, S.; Seif, M.; Asayesh, E.J.; Bernard, F.; Hassani, B.; Li, T. Enhanced salt tolerance and photosynthetic performance: Implication of $\gamma$-amino butyric acid application in salt-exposed lettuce (Lactuca sativa L.) plants. Plant Physiol. Bioch. 2018, 130, 157-172. [CrossRef] [PubMed]

15. Ramesh, S.A.; Tyerman, S.D.; Xu, B.; Bose, J.; Kaur, S.; Conn, V.; Domingos, P.; Ullah, S.; Wege, S.; Shabala, S.; et al. GABA signalling modulates plant growth by directly regulating the activity of plant-specific anion transporters. Nat. Commun. 2015, 6, 7879. [CrossRef] [PubMed]

16. Takayama, M.; Matsukura, C.; Ariizumi, T.; Ezura, H. Activating glutamate decarboxylase activity by removing the autoinhibitory domain leads to hyper $\gamma$-aminobutyric acid (GABA) accumulation in tomato fruit. Plant Cell Rep. 2017, 36, 103-116. [CrossRef]

17. Xie, T.; Ji, J.; Chen, W.; Yue, J.; Du, C.; Sun, J.; Chen, L.; Jiang, Z.; Shi, S. GABA negatively regulates adventitious root development. J. Exp. Bot. 2019, 71, 1459-1474. [CrossRef]

18. Yue, J.Y.; Du, C.J.; Ji, J.; Xie, T.T.; Chen, W.; Chang, E.M.; Chen, L.Z.; Jiang, Z.P.; Shi, S.Q. Inhibition of $\alpha$-ketoglutarate dehydrogenase activity affects adventitious root growth in poplar via changes in GABA shunt. Planta 2018, 248, 963-979. [CrossRef]

19. Bouche, N.; Fromm, H. GABA in plants: Just a metabolite? Trends Plant Sci. 2004, 9, 110-115. [CrossRef]

20. Fait, A.; Fromm, H.; Walter, D.; Galili, G.; Fernie, A.R. Highway or byway: The metabolic role of the GABA shunt in plants. Trends Plant Sci. 2008, 13, 14-19. [CrossRef] [PubMed]

21. Ramesh, S.A.; Kamran, M.; Sullivan, W.; Chirkova, L.; Okamoto, M.; Degryse, F.; McLaughlin, M.; Gilliham, M.; Tyerman, S.D. Aluminum-activated malate transporters can facilitate GABA transport. Plant Cell 2018, 30, 1147-1164. [CrossRef]

22. Okumoto, S.; Pilot, G. Amino acid export in plants: A missing link in nitrogen cycling. Mol. Plant 2011, 4, 453-463. [CrossRef] [PubMed]

23. Tegeder, M. Transporters for amino acids in plant cells: Some functions and many unknowns. Curr. Opin. Plant Biol. 2012, 15, 315-321. [CrossRef]

24. Schwacke, R.; Grallath, S.; Breitkreuz, K.E.; Stransky, E.; Stransky, H.; Frommer, W.B.; Rentsch, D. LeProT1, a Transporter for Proline, Glycine Betaine, and $\gamma$-Amino Butyric Acid in Tomato Pollen. Plant Cell 1999, 11, 377-391. [PubMed] 
25. Meyer, A.; Eskandari, S.; Grallath, S.; Rentsch, D. AtGAT1, a high affinity transporter for $\gamma$-aminobutyric acid in Arabidopsis thaliana. J. Biol. Chemstry 2006, 281, 7197-7204. [CrossRef] [PubMed]

26. Bai, X.; Xu, J.; Shao, X.; Luo, W.; Niu, Z.; Gao, C.; Wan, D. A Novel Gene Coding $\gamma$-Aminobutyric Acid Transporter May Improve the Tolerance of Populus euphratica to Adverse Environments. Front. Plant Sci. 2019, 10, 1083. [CrossRef]

27. Ma, J.; Wan, D.; Duan, B.; Bai, X.; Bai, Q.; Chen, N.; Ma, T. Genome sequence and genetic transformation of a widely distributed and cultivated poplar. Plant Biotechnol. J. 2019, 17, 451-460. [CrossRef]

28. Ma, T.; Wang, J.; Zhou, G.; Yue, Z.; Hu, Q.; Chen, Y.; Liu, B.; Qiu, Q.; Wang, Z.; Zhang, J.; et al. Genomic insights into salt adaptation in a desert poplar. Nat. Commun. 2013, 4, 2797. [CrossRef]

29. Daccord, N.; Celton, J.M.; Linsmith, G.; Becker, C.; Choisne, N.; Schijlen, E.; van de Geest, H.; Bianco, L.; Micheletti, D.; Velasco, R.; et al. High-quality de novo assembly of the apple genome and methylome dynamics of early fruit development. Nat. Genet. 2017, 49, 1099-1106. [CrossRef]

30. Jung, S.; Lee, T.; Cheng, C.H.; Buble, K.; Zheng, P.; Yu, J.; Humann, J.; Ficklin, S.P.; Gasic, K.; Scott, K.; et al. 15 years of GDR: New data and functionality in the Genome Database for Rosaceae. Nucleic Acids Res. 2019, 47, D1137-D1145. [CrossRef]

31. Chen, C.; Xia, R.; Chen, H.; He, Y. TBtools, a Toolkit for Biologists integrating various HTS-data handling tools with a user-friendly interface. bioRxiv 2018, 289660. [CrossRef]

32. Bailey, T.L.; Boden, M.; Buske, F.A.; Frith, M.; Grant, C.E.; Clementi, L.; Ren, J.; Li, W.W.; Noble, W.S. MEME SUITE: Tools for motif discovery and searching. Nucleic Acids Res. 2009, 37, W202-W208. [CrossRef] [PubMed]

33. Lescot, M.; Dehais, P.; Thijs, G.; Marchal, K.; Moreau, Y.; VandePeer, Y. PlantCARE, a database of plant cis-acting regulatory elements and a portal to tools for in silico analysis of promoter sequences. Nucleic Acids Res. 2002, 30, 325-327. [CrossRef]

34. Chen, C.; Chen, H.; Zhang, Y.; Thomas, H.R.; Frank, M.H.; He, Y.; Xia, R. TBtools: An Integrative Toolkit Developed for Interactive Analyses of Big Biological Data. Mol. Plant 2020, 13, 1194-1202. [CrossRef]

35. Ma, B.; Yuan, Y.; Gao, M.; Qi, T.; Li, M.; Ma, F. Genome-wide identification, molecular evolution, and expression divergence of aluminum-activated malate transporters in apples. Int. J. Mol. Sci. 2018, 19, 2807. [CrossRef]

36. Bergmann, R.; Kongsbak, K.; Sorensen, P.L.; Sander, T.; Balle, T. A unified model of the GABA A receptor comprising agonist and benzodiazepine binding sites. PLoS ONE 2013, 8, e52323. [CrossRef] [PubMed]

37. Miller, P.S.; Aricescu, A.R. Crystal structure of a human GABA $\mathrm{A}$ receptor. Nature 2014, 512, 270-275. [CrossRef]

38. Velasco, R.; Zharkikh, A.; Affourtit, J.; Dhingra, A.; Cestaro, A.; Kalyanaraman, A.; Fontana, P.; Bhatnagar, S.K.; Troggio, M.; Pruss, D.; et al. The genome of the domesticated apple (Malus $x$ domestica Borkh.). Nat. Genet. 2010, 42, 833-839. [CrossRef]

39. Da, L.; Liu, Y.; Yang, J.; Tian, T.; She, J.; Ma, X.; Xu, W.; Su, Z. AppleMDO: A Multi-Dimensional Omics Database for Apple Co-Expression Networks and Chromatin States. Front. Plant Sci. 2019, 10, 1333. [CrossRef]

40. Valentinuzzi, F.; Venuti, S.; Pii, Y.; Marroni, F.; Cesco, S.; Hartmann, F.; Mimmo, T.; Morgante, M.; Pinton, R.; Tomasi, N.; et al. Common and specific responses to iron and phosphorus deficiencies in roots of apple tree (Malus $x$ domestica). Plant Mol. Biol. 2019, 101, 129-148. [CrossRef]

41. Che-Othman, M.H.; Jacoby, R.P.; Millar, A.H.; Taylor, N.L. Wheat mitochondrial respiration shifts from the tricarboxylic acid cycle to the GABA shunt under salt stress. New Phytol. 2020, 225, 1166-1180. [CrossRef] [PubMed]

42. Liu, X.; Hu, X.-M.; Jin, L.-F.; Shi, C.-Y.; Liu, Y.-Z.; Peng, S.-A. Identification and transcript analysis of two glutamate decarboxylase genes, CsGAD1 and CsGAD2, reveal the strong relationship between CsGAD1 and citrate utilization in citrus fruit. Mol. Biol. Rep. 2014, 41, 6253-6262. [CrossRef] [PubMed]

43. Bouche, N.; Fait, A.; Zik, M.; Fromm, H. The root-specific glutamate decarboxylase (GAD1) is essential for sustaining GABA levels in Arabidopsis. Plant Mol. Biol. 2004, 55, 315-325. [CrossRef] [PubMed]

44. Zhuang, Y.; Ren, G.; He, C.; Li, X.; Meng, Q.; Zhu, C.; Wang, R.; Zhang, J. Cloning and Characterization of a Maize cDNA Encoding Glutamate Decarboxylase. Plant Mol. Biol. Repot. 2010, 28, 620-626. [CrossRef]

45. Trobacher, C.P.; Zarei, A.; Liu, J.; Clark, S.M.; Bozzo, G.G.; Shelp, B.J. Calmodulin-dependent and calmodulinindependent glutamate decarboxylases in apple fruit. BMC Plant Biol. 2013, 13, 144. [CrossRef] [PubMed]

46. Mei, X.; Chen, Y.; Zhang, L.; Fu, X.; Wei, Q.; Grierson, D.; Zhou, Y.; Huang, Y.; Dong, F.; Yang, Z. Dual mechanisms regulating glutamate decarboxylases and accumulation of $\gamma$-aminobutyric acid in tea (Camellia sinensis) leaves exposed to multiple stresses. Sci. Rep. 2016, 6, 23685. [CrossRef]

47. Ji, J.; Zheng, L.; Yue, J.; Yao, X.; Chang, E.; Xie, T.; Deng, N.; Chen, L.; Huang, Y.; Jiang, Z.; et al. Identification of two CiGADs from Caragana intermedia and their transcriptional responses to abiotic stresses and exogenous abscisic acid. PeerJ 2017, 5, e3439. [CrossRef]

48. Akama, K.; Akihiro, T.; Kitagawa, M.; Takaiwa, F. Rice (Oryza sativa) contains a novel isoform of glutamate decarboxylase that lacks an authentic calmodulin-binding domain at the C-terminus. Biochim. Biophys. Acta. 2001, 1522, 143-150. [CrossRef]

49. Sharma, T.; Dreyer, I.; Kochian, L.; Pineros, M.A. The ALMT Family of Organic Acid Transporters in Plants and Their Involvement in Detoxification and Nutrient Security. Front. Plant Sci. 2016, 7, 1488. [CrossRef]

50. Hedrich, R.; Salvador-Recatala, V.; Dreyer, I. Electrical Wiring and Long-Distance Plant Communication. Trends Plant Sci. 2016, 21, 376-387. [CrossRef]

51. Barbier-Brygoo, H.; De Angeli, A.; Filleur, S.; Frachisse, J.M.; Gambale, F.; Thomine, S.; Wege, S. Anion channels/transporters in plants: From molecular bases to regulatory networks. Annu. Rev. Plant Biol. 2011, 62, 25-51. [CrossRef] [PubMed] 